$12-1-1998$

\title{
Excitation Efficiency of a Morphology-Dependent Resonance by a Focused Gaussian Beam
}

James A. Lock

Cleveland State University, j.lock@csuohio.edu

Follow this and additional works at: https://engagedscholarship.csuohio.edu/sciphysics_facpub

Part of the Physics Commons

How does access to this work benefit you? Let us know!

\section{Publisher's Statement}

This paper was published in Journal of the Optical Society of America A: Optics Image Science and Vision and is made available as an electronic reprint with the permission of OSA. The paper can be found at the following URL on the OSA website: http://www.opticsinfobase.org/josaa/ abstract.cfm?URI=josaa-15-12-2986. Systematic or multiple reproduction or distribution to multiple locations via electronic or other means is prohibited and is subject to penalties under law.

\section{Original Citation}

Lock, James A. "Excitation Efficiency of a Morphology-Dependent Resonance by a Focused Gaussian Beam." Journal of the Optical Society of America A: Optics Image Science and Vision 15 (1998): 2986-2994.

\section{Repository Citation}

Lock, James A., "Excitation Efficiency of a Morphology-Dependent Resonance by a Focused Gaussian Beam" (1998). Physics Faculty Publications. 28.

https://engagedscholarship.csuohio.edu/sciphysics_facpub/28

This Article is brought to you for free and open access by the Physics Department at EngagedScholarship@CSU. It has been accepted for inclusion in Physics Faculty Publications by an authorized administrator of EngagedScholarship@CSU. For more information, please contact library.es@csuohio.edu. 


\title{
Excitation efficiency of a morphology-dependent resonance by a focused Gaussian beam
}

\author{
James A. Lock \\ Department of Physics, Cleveland State University, Cleveland, Ohio 44115
}

Received June 17, 1998; revised manuscript received August 21, 1998; accepted September 1, 1998

\begin{abstract}
The excitation efficiency of a morphology-dependent resonance (MDR) by an incident beam is defined as the fraction of the beam power channeled into the MDR. The efficiency is calculated for a focused Gaussian beam of arbitrary width incident on either a spherical particle or a cylindrical fiber located at an arbitrary position in the plane of the beam waist. In each case a simple formula for the efficiency is derived by use of the localized approximation for the beam-shape coefficients in the partial-wave expansion of the beam. The physical interpretation of the efficiency formulas is also discussed. () 1998 Optical Society of America [S0740-3232(98)01512-9]
\end{abstract}

OCIS codes: $290.0290,290.4020$.

\section{INTRODUCTION}

When a beam of electromagnetic radiation is incident on a small dielectric particle of high symmetry, such as a sphere or a circular cylinder, a morphology-dependent resonance (MDR) is excited in one of the partial waves in the partial-wave expansion of the incident beam at certain combinations of the wavelength of the radiation and the particle's radius and refractive index. According to van de Hulst's localization principle ${ }^{1}$ the resonant partial wave corresponds to an incident light ray whose impact parameter with respect to the center of the particle is somewhat larger than the particle's radius, and therefore the ray would classically pass the particle without striking it. The fact that MDR's are excited by rays that miss the particle is paradoxical in ray optics, but it is easily understood in wave optics. A portion of the partial wave evanescently tunnels through the centrifugal barrier surrounding the particle and is transmitted to the particle's interior. $^{2-4}$ Once inside, the partial wave is trapped in the particle's radial interior potential well and successively reflects back and forth within it. At resonance, approximately an integer number of half-wavelengths of the partial wave fit within the radial potential well, and the successive internal reflections are in phase with one another. As a result, a large energy density builds up inside the particle just beneath its surface, ${ }^{5,6}$ and at each internal reflection a certain percentage of the interior field tunnels back out through the centrifugal barrier and is detected in the far zone as a resonant amplification of the scattered light. ${ }^{7,8}$

A decade ago it was experimentally observed ${ }^{9,10}$ that the efficiency of MDR excitation depends on whether the incident beam is a plane wave or a focused Gaussian beam, and for Gaussian beam incidence it depends on the degree of beam focusing and the position of the beam's focal waist with respect to the particle. This dependence of the MDR excitation efficiency on the properties of the beam is intuitively sensible since a large percentage of the corresponding rays in a tightly focused beam ${ }^{11}$ incident somewhat beyond the particle's edge have the cor- rect impact parameter for MDR generation, whereas most of the rays in a loosely focused beam or a plane wave do not.

Numerical computations of the absorption efficiency, ${ }^{12}$ the interior source function, ${ }^{12,13}$ and the total energy inside a spherical particle $\mathrm{e}^{13,14}$ as a function of the width of the illuminating beam and the beam's focal waist position have corroborated the experimental observations. In addition, an analytical approximation for the position of the center of the beam's focal waist that gives the largest MDR excitation efficiency (i.e., the optimal position of the focal waist) has been derived ${ }^{15}$ by use of the localized approximation for the beam-shape coefficients in the partial-wave expansion of a focused Gaussian beam ${ }^{16-18}$ and has been compared with the results of numerical computations for sphere MDR's. Also, a simple physically based ray-theory approximation to the MDR excitation efficiency at the optimal position of the beam's focal waist has been derived. ${ }^{19}$

In this paper we derive an analytical approximation to the MDR excitation efficiency for a focused Gaussian beam of arbitrary width incident on a sphere (Section 2) or upon a circular cylinder (Section 3) located at an arbitrary position in the plane of the beam waist, using the localized approximation beam-shape coefficients. We discuss the limitations of our results in Section 4 . The expressions for the excitation efficiency derived here, along with the previously derived expression for the optimal beam position, ${ }^{15,20}$ completely characterize the dependence of the MDR excitation efficiency on the properties of an incident Gaussian beam.

\section{MORPHOLOGY-DEPENDENT- RESONANCE EXCITATION EFFICIENCY FOR A SPHERICAL PARTICLE}

We consider an electromagnetic beam with peak electric field strength $E_{0}$, wavelength $\lambda$, and wave number $k$ $=2 \pi / \lambda$ traveling parallel to the $z$ axis and incident upon a nonabsorbing dielectric spherical particle of radius $a$ and real refractive index $n$ whose center is at the origin of 
coordinates. This geometry is shown in Fig. 1. The beam-shape coefficients in the partial-wave expansion of the incident beam are $A_{l, m}$ and $B_{l, m}$, where $l$ is the partial-wave number $(1 \leqslant l<\infty)$ and $m$ is the azimuthal mode number $(-l \leqslant m \leqslant l)$. When the spherical Bessel functions in the partial-wave expansion are decomposed into incoming and outgoing parts and the outgoing part is evaluated in the far zone, the beam power may be expressed in terms of the beam-shape coefficients as

$$
\begin{aligned}
P_{\text {beam }}= & \left(\frac{E_{0}^{2}}{2 \mu_{0} c}\right)\left(\frac{\pi}{4 k^{2}}\right) \sum_{l=1}^{\infty} \frac{2 l+1}{l(l+1)} \sum_{m=-l}^{l} \frac{(l+|m|) !}{(l-|m|) !} \\
& \times\left(\left|A_{l, m}\right|^{2}+\left|B_{l, m}\right|^{2}\right),
\end{aligned}
$$

where $\mu_{0}$ is the permeability of free space and $c$ is the speed of light. The beam is scattered by a spherical particle, and the scattered power is ${ }^{21}$

$$
\begin{aligned}
P_{\text {scatt }}= & \left(\frac{E_{0}^{2}}{2 \mu_{0} c}\right)\left(\frac{\pi}{k^{2}}\right) \sum_{l=1}^{\infty} \frac{2 l+1}{l(l+1)} \sum_{m=-l}^{l} \frac{(l+|m|) !}{(l-|m|) !} \\
& \times\left(\left|A_{l, m}\right|^{2}\left|a_{l}\right|^{2}+\left|B_{l, m}\right|^{2}\left|b_{l}\right|^{2}\right),
\end{aligned}
$$

where $a_{l}$ and $b_{l}$ are the partial-wave scattering amplitudes.

We assume that as $\lambda$ is varied while $a$ and $n$ are held fixed, the particle size parameter $x=2 \pi a / \lambda$ sweeps through a TE-polarized or a TM-polarized MDR of linewidth $2 \Gamma$ in partial wave $L$. Since there is no absorption of light by the particle and $n$ is assumed to be real, the partial-wave scattering amplitudes satisfy ${ }^{7} \quad\left|b_{L}\right|^{2}$ $=1.0$ for a TE resonance and $\left|a_{L}\right|^{2}=1.0$ for a TM resonance at the value of $\lambda$ that corresponds to the center of the resonance linewidth. Once radiative equilibrium between the incident beam and the particle has been reached and the energy stored in the MDR mode within the particle is constant, the incident energy per second coupled into the MDR is equal to the energy per second tunneling out of the MDR and appearing in the far zone as scattered light. Thus we define the TE-polarized and

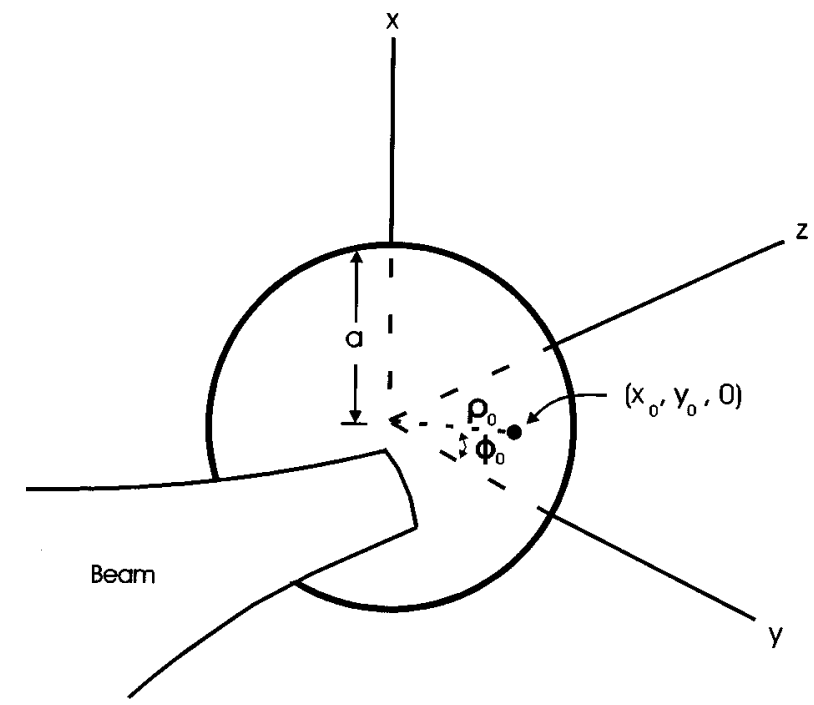

Fig. 1. Focused Gaussian beam with the center of its focal waist at $\left(x_{0}, y_{0}, 0\right)$ incident on a spherical particle of radius $a$ whose center is at the origin of coordinates.
TM-polarized MDR excitation efficiencies $\epsilon_{L}^{\mathrm{TE}}$ and $\epsilon_{L}^{\mathrm{TM}}$, respectively, as the ratio of the power elastically scattered by the MDR mode divided by the incident beam power:

$$
\begin{aligned}
& \epsilon_{L}^{\mathrm{TE}}=P_{\text {scatt, } L}^{\mathrm{TE}} / P_{\text {beam }}, \\
& \epsilon_{L}^{\mathrm{TM}}=P_{\text {scatt, } L}^{\mathrm{TM}} / P_{\text {beam }},
\end{aligned}
$$

where we obtain $P_{\mathrm{scatt}, L}^{\mathrm{TE}}$ and $P_{\mathrm{scatt}, L}^{\mathrm{TM}}$ from Eq. (2) by considering only the resonant partial wave and employing the value 1.0 for the appropriate partial-wave scattering amplitude at the center of the resonance linewidth; i.e.,

$$
\begin{aligned}
P_{\mathrm{scatt}, L}^{\mathrm{TE}}= & \left(\frac{E_{0}^{2}}{2 \mu_{0} c}\right)\left(\frac{\pi}{k^{2}}\right) \frac{2 L+1}{L(L+1)} \\
& \times \sum_{m=-L}^{L} \frac{(L+|m|) !}{(L-|m|) !}\left|B_{L, m}\right|^{2}, \\
P_{\mathrm{scatt}, L}^{\mathrm{TM}}= & \left(\frac{E_{0}^{2}}{2 \mu_{0} c}\right)\left(\frac{\pi}{k^{2}}\right) \frac{2 L+1}{L(L+1)} \\
& \times \sum_{m=-L}^{L} \frac{(L+|m|) !}{(L-|m|) !}\left|A_{L, m}\right|^{2} .
\end{aligned}
$$

We now specify our results to a focused Gaussian beam of electric field 1/e half-width $w_{0}$ positioned such that the center of its focal waist is located at $\left(x_{0}, y_{0}, 0\right)$ as in Fig. 1. The localized approximation beam-shape coefficients provide an accurate approximation to both the amplitude profile and the phase fronts of the Gaussian beam as long as the beam's focal waist is wider than a few wavelengths. The localized coefficients for scattering by a sphere are based on the Davis-Barton model of Gaussian beams and are expressed in spherical coordinates as ${ }^{15}$

$$
\begin{aligned}
& A_{l, 0}=2 i\left[\frac{l(l+1)}{\left(l+\frac{1}{2}\right)}\right] \cos \phi_{0} \\
& \times \exp \left\{-\left[\frac{\rho_{0}^{2}}{w_{0}^{2}}+s^{2}\left(l+\frac{1}{2}\right)^{2}\right]\right\} I_{1}(Q), \\
& A_{l, \pm m}=\left[-i \exp \left(\mp i \phi_{0}\right) /\left(l+\frac{1}{2}\right)\right]^{|m|-1} \\
& \times \exp \left\{-\left[\frac{\rho_{0}^{2}}{w_{0}^{2}}+s^{2}\left(l+\frac{1}{2}\right)^{2}\right]\right\} \\
& \times\left[I_{|m|-1}(Q)+\exp \left(\mp i 2 \phi_{0}\right) I_{|m|+1}(Q)\right] ; \\
& B_{l, 0}=2 i\left[\frac{l(l+1)}{\left(l+\frac{1}{2}\right)}\right] \sin \phi_{0} \\
& \times \exp \left\{-\left[\frac{\rho_{0}^{2}}{w_{0}^{2}}+s^{2}\left(l+\frac{1}{2}\right)^{2}\right]\right\} I_{1}(Q), \\
& B_{l, \pm m}=\left(\frac{ \pm 1}{i}\right)\left[-i \exp \left(\mp i \phi_{0}\right) /\left(l+\frac{1}{2}\right)\right]^{|m|-1} \\
& \times \exp \left\{-\left[\frac{\rho_{0}^{2}}{w_{0}^{2}}+s^{2}\left(l+\frac{1}{2}\right)^{2}\right]\right\} \\
& \times\left[I_{|m|-1}(Q)-\exp \left(\mp 2 i \phi_{0}\right) I_{|m|+1}(Q)\right],
\end{aligned}
$$


where $I_{l}(Q)$ are modified Bessel functions of the first kind, the beam's focal waist position is

$$
\begin{aligned}
& x_{0}=\rho_{0} \cos \phi_{0}, \\
& y_{0}=\rho_{0} \sin \phi_{0}, \\
& z_{0}=0,
\end{aligned}
$$

the beam's confinement parameter $s$ is

$$
s=1 / k w_{0},
$$

and the argument of the modified Bessel function is

$$
Q=2 s\left(l+\frac{1}{2}\right) \rho_{0} / w_{0} .
$$

If the beam were focused either before or after the particle, i.e., if $z_{0} \neq 0, Q$ would be a complex number, and the analysis of the excitation efficiency would become considerably more complicated.

For a typical partial wave $l \gg 1$ in a typical focused Gaussian beam, ${ }^{15}$ the magnitude of $A_{l, m}$ and $B_{l, m}$ reaches its peak value when $m \approx 0$ and decreases rapidly for large $|m|$. Thus the approximation

$$
\frac{(l+|m|) !}{(l-|m|) !} \approx\left(l+\frac{1}{2}\right)^{|2 m|},
$$

which is most accurate when $l$ is large and $|m|$ is small, may be substituted along with Eqs. (7) for the beamshape coefficients into Eqs. (1), (5), and (6). With this approximation, the addition formulas for modified Bessel functions $^{22}$

$$
I_{P}(2 x)=\sum_{n=-\infty}^{\infty} I_{n}(x) I_{p-n}(x)
$$

for $p=0,2$ then allow the sum over $m$ in Eqs. (1), (5), and (6) to be evaluated, giving

$$
\begin{aligned}
& \sum_{m} \frac{(L+|m|) !}{(L-|m|) !}\left|A_{L, m}\right|^{2} \\
& \approx 2\left(L+\frac{1}{2}\right)^{2} \exp \left\{-2\left[\frac{\rho_{0}^{2}}{w_{0}{ }^{2}}+s^{2}\left(L+\frac{1}{2}\right)^{2}\right]\right\} \\
& \times\left[I_{0}(2 Q)+\cos 2 \phi_{0} I_{2}(2 Q)\right], \\
& \sum_{m} \frac{(L+|m|) !}{(L-|m|) !}\left|B_{L, m}\right|^{2} \\
& \approx 2\left(L+\frac{1}{2}\right)^{2} \exp \left\{-2\left[\frac{\rho_{0}{ }^{2}}{w_{0}{ }^{2}}+s^{2}\left(L+\frac{1}{2}\right)^{2}\right]\right\} \\
& \times\left[I_{0}(2 Q)-\cos 2 \phi_{0} I_{2}(2 Q)\right] \text {. }
\end{aligned}
$$

In applying the addition formulas for modified Bessel functions to Eqs. (1), (5), and (6) we changed the sum over $m$ from $-L \leqslant m \leqslant L$ to $-\infty<m<\infty$. This change should not cause a problem because, as was mentioned above, the numerical value of $A_{l m}$ and $B_{l m}$ decreases rapidly for large $|m|$. The use of the addition formulas for modified Bessel functions in relations (13) has not been considered previously and greatly simplifies the calculations of excitation efficiency for sphere MDR's.
As a consistency check of these approximations, when relations (13) are substituted into Eq. (1) the incident beam power becomes

$$
\begin{aligned}
P_{\text {beam }} \approx & \left(\frac{E_{0}^{2}}{2 \mu_{0} c}\right)\left(\frac{2 \pi}{k^{2}}\right) \exp \left(-2 \rho_{0}^{2} / w_{0}^{2}\right) \sum_{l=1}^{\infty}\left(l+\frac{1}{2}\right) \\
& \times I_{0}\left[4 s\left(l+\frac{1}{2}\right) \rho_{0} / w_{0}\right] \exp \left[-2 s^{2}\left(l+\frac{1}{2}\right)^{2}\right] .
\end{aligned}
$$

If many partial waves contribute to the partial-wave expansion of the beam, relation (14) can be converted approximately into an integral over the impact parameter, to give $^{23}$

$$
\begin{aligned}
P_{\text {beam }} \approx & \left(\frac{E_{0}{ }^{2}}{2 \mu_{0} c}\right)\left(\frac{2 \pi}{k^{2}}\right) \exp \left(-2 \rho_{0}^{2} / w_{0}^{2}\right) \int_{0}^{\infty} l \mathrm{~d} l \\
& \times I_{0}\left(4 s l \rho_{0} / w_{0}\right) \exp \left(-2 s^{2} l^{2}\right) \\
= & \left(\frac{E_{0}^{2}}{2 \mu_{0} c}\right)\left(\frac{\pi w_{0}^{2}}{2}\right),
\end{aligned}
$$

as expected for a focused Gaussian beam with electric field 1/e half-width $w_{0}$ in the Fresnel diffraction model of beam propagation. ${ }^{24}$

We can now evaluate the MDR excitation efficiency by combining relations (3)-(6), (13), and (15) to give

$$
\begin{aligned}
\epsilon_{L}^{\mathrm{TE}}= & 8\left(L+\frac{1}{2}\right) s^{2} \exp \left\{-2\left[\frac{\rho_{0}^{2}}{w_{0}^{2}}+s^{2}\left(L+\frac{1}{2}\right)^{2}\right]\right\} \\
& \times\left[I_{0}(2 Q)-\cos 2 \phi_{0} I_{2}(2 Q)\right], \\
\epsilon_{L}^{\mathrm{TM}}= & 8\left(L+\frac{1}{2}\right) s^{2} \exp \left\{-2\left[\frac{\rho_{0}^{2}}{w_{0}^{2}}+s^{2}\left(L+\frac{1}{2}\right)^{2}\right]\right\} \\
& \times\left[I_{0}(2 Q)+\cos 2 \phi_{0} I_{2}(2 Q)\right] .
\end{aligned}
$$

Equations (16) are valid for both arbitrary $w_{0}$ and arbitrary transverse positioning of the beam-waist center $\left(x_{0}, y_{0}, 0\right)$ with respect to the spherical particle. From a numerical-method point of view, only two modified Bessel functions, for which various approximations are known, ${ }^{15,25}$ are to be evaluated in Eqs. (16). On the other hand, in previous numerical computations for sphere MDR's produced by an off-axis Gaussian beam, the beamshape coefficients had to be computed and the sum over $m$ had to be computed and tested for convergence. Also, since Eqs. (16) are based on Lorenz-Mie theory, they represent an improvement over the intuitive but approximate ray theory result of Ref. 19 .

If the beam strikes the spherical particle approximately on center, i.e., $\rho_{0} \approx 0$, then $Q$ is small and the modified Bessel functions in Eqs. (16) may be Taylorseries expanded. ${ }^{26}$ The excitation efficiency for TE- and TM-polarized MDR's then becomes

$$
\begin{aligned}
\epsilon_{L}^{\mathrm{TE}} \approx \epsilon_{L}^{\mathrm{TM}} \approx & 8\left(L+\frac{1}{2}\right) s^{2} \exp \left[-2 s^{2}\left(L+\frac{1}{2}\right)^{2}\right] \\
& \times\left[1+O\left(Q^{2}\right)\right] .
\end{aligned}
$$

The maximum value of relation (17) occurs when

$$
2 s^{2}\left(L+\frac{1}{2}\right)^{2}=1,
$$


or, since low radial-order resonances occur in the partial waves $L \lesssim n k a$, the beam width for maximum on-axis MDR excitation is

$$
W_{0}=\lambda L /(2)^{1 / 2} \pi \approx(2)^{1 / 2} n a
$$

and the maximum on-axis efficiency is

$$
\left(\epsilon_{L}\right)_{\max } \approx 4 e^{-1 / L} \approx 0.234 \lambda / n a .
$$

Since high-radial-order MDR's occur for $L \gtrsim k a$, the factor $n$ should be deleted in relations (19) and (20) when one describes high-radial-order resonances.

If the beam strikes the spherical particle far off axis, e.g., $\rho_{0} \approx a$, then $Q$ is large and the modified Bessel functions in Eqs. (16) may be approximated by their asymptotic expansions. ${ }^{27}$ The MDR excitation efficiencies then become

$$
\begin{aligned}
\epsilon_{L}^{\mathrm{TE}} \approx & \frac{4 s^{2}\left(L+\frac{1}{2}\right)}{(\pi Q)^{1 / 2}} \\
& \times \exp \left\{-2\left[\frac{\rho_{0}}{w_{0}}-s\left(L+\frac{1}{2}\right)\right]^{2}\right\}\left[\left(1-\cos 2 \phi_{0}\right)\right. \\
& \left.+\frac{1}{16 Q}\left(1+15 \cos 2 \phi_{0}\right)+O\left(\frac{1}{Q^{2}}\right)\right] \\
\epsilon_{L}^{\mathrm{TM}} \approx & \frac{4 s^{2}\left(L+\frac{1}{2}\right)}{(\pi Q)^{1 / 2}} \\
& \times \exp \left\{-2\left[\frac{\rho_{0}}{w_{0}}-s\left(L+\frac{1}{2}\right)\right]^{2}\right\}\left[\left(1+\cos 2 \phi_{0}\right)\right. \\
& \left.+\frac{1}{16 Q}\left(1-15 \cos 2 \phi_{0}\right)+O\left(\frac{1}{Q^{2}}\right)\right] .
\end{aligned}
$$

Relations (21) and (22) illustrate that TM-polarized resonances are most efficiently excited when $\phi_{0}=0^{\circ}, 180^{\circ}$, TE-polarized resonances are most efficiently excited when $\phi_{0}=90^{\circ}, 270^{\circ}$, and the highest-efficiency beam position is

$$
\rho_{0} / w_{0}=s\left(L+\frac{1}{2}\right) .
$$

The optimum $\rho_{0}$ in Eq. (23) has previously been expressed in terms of $n$, the particle size parameter at resonance $x_{\text {res }}$, and the radial order number $i$ of the MDR in Eq. (63) of Ref. 15. At the optimal off-axis beam position the TE or TM excitation efficiency is

$$
\epsilon_{L}^{\mathrm{TE}} \approx \epsilon_{L}^{\mathrm{TM}} \approx\left(\frac{2}{\pi}\right)^{3 / 2} \frac{\lambda}{w_{0}} \approx 0.508 \frac{\lambda}{w_{0}} .
$$

This result is compared with the value $\epsilon_{L}^{\mathrm{TE}} \approx \epsilon_{L}^{\mathrm{TM}}$ $\approx 0.143 \lambda / w_{0}$ obtained by use of ray theory in Ref. 19 . The ray-theory result is seen here to give the correct order of magnitude and the correct dependence on $\lambda$ and $w_{0}$. The maximum MDR excitation efficiency of relation (24) is independent of the partial-wave number $L$, of whether the resonance is TE or TM polarized, and of the resonance half-width $\Gamma$, assuming that beam wavelength $\lambda$ corresponds to the center of the MDR linewidth.

The derivation leading to relation (24) ignored the finite linewidth $\Delta \lambda_{\text {beam }}$ of the laser beam. If the laser line- width exceeds that of the resonance, only some of the wavelengths within the laser linewidth are able to excite the MDR. Such is the case for low-radial-order MDR's, for which $\Delta \lambda_{\mathrm{MDR}}$ can easily ${ }^{28}$ be less than $10^{-5} \mathrm{~nm}$, whereas a typical laser linewidth is ${ }^{29} \Delta \lambda_{\text {beam }}$ $\approx 10^{-3} \mathrm{~nm}$. If the size parameter interval encompassed by the laser beam's linewidth is

$$
2 \gamma=x \Delta \lambda_{\text {beam }} / \lambda
$$

and the laser beam power as a function of $\lambda$ is modeled as a Gaussian centered on the dominant laser wavelength, relation (24) should be multiplied by the half-width ratio $\Gamma /\left(\Gamma^{2}+\gamma^{2}\right)^{1 / 2}$. For a wide-resonance (high-radial-order) or a narrow-linewidth laser, the ratio is unity and relation (24) remains unchanged. For a narrow-resonance (low-radial-order) or a broad-linewidth laser, the ratio approaches $\Gamma / \gamma$. The low value of this ratio is the cause of the small excitation efficiency of low-radial-order MDR's and their resulting absence in elastic scattering spectra.

A simple example illustrating the dependence of the MDR excitation efficiency on the beam width and position is as follows: A spherical particle of radius $a$ $\approx 22.5 \mu \mathrm{m}$ can exhibit MDR's in partial waves of $L$ $\approx 300$ when it is illuminated by laser light with $\lambda$ $=0.5145 \mu \mathrm{m}$. We assume that the beam has on-axis incidence, i.e., $\rho_{0}=0 \mu \mathrm{m}$, and that it is minimally focused to $w_{0}=1000 \mu \mathrm{m}$, approximating a plane wave striking the particle. If $\lambda, a$, and $n$ are suitably related so as to excite an $L \approx 300$ resonance and the laser linewidth satisfies $\gamma \ll \Gamma$, the fraction of the incident beam power coupled into the MDR is $\epsilon_{300}^{\mathrm{TE}}=\epsilon_{300}^{\mathrm{TM}}=1.6 \times 10^{-5}$. The excitation efficiency is small since most of the incident rays in the wide beam do not have the correct impact parameter for exciting the resonance. If the beam is now focused to $w_{0}=8 \mu \mathrm{m}$ (approximately $1 / 3$ of the particle radius) and is still incident on axis, the MDR excitation efficiency falls to $\epsilon_{300}^{\mathrm{TE}}=\epsilon_{300}^{\mathrm{TM}}=3.6 \times 10^{-9}$ since only the tail of the Gaussian beam has the proper impact parameter for exciting the MDR. If, however, the $w_{0}=8 \mu \mathrm{m}$ beam is moved to the optimal position $\rho_{0}=24.6 \mu \mathrm{m}$ either on the $x$ axis so as to excite primarily a TM-polarized resonance or on the $y$ axis so as to excite primarily a TEpolarized resonance, then $\epsilon_{300}=3.3 \times 10^{-2}$ of the incident beam power is coupled into the TE or the TM resonance, assuming no absorption. This large efficiency is impressive for a beam that classically misses the particle, and it attests to the efficiency of the evanescent tunneling mechanism at resonance.

\section{MORPHOLOGY-DEPENDENT RESONANCE EXCITATION EFFICIENCY FOR A CYLINDRICAL FIBER}

When a Gaussian beam excites a MDR in a long nonabsorbing dielectric fiber of radius $a$ and real refractive index $n$, the beam-fiber geometry renders the situation more complicated than for sphere MDR's. Physically, scattering by a cylinder depends strongly on the angle $\xi$ that the incident beam makes with the normal to the cylinder axis. ${ }^{30}$ On the other hand, when a beam is incident on a sphere, a rotated coordinate system $x^{\prime} y^{\prime} z^{\prime}$ can always be found in which the beam propagates parallel to 
the $z^{\prime}$ axis, as in Fig. 1. Mathematically, the different symmetries of the beam-sphere and beam-cylinder geometries are reflected in the respective eigenfunctions and eigenvalues of the scalar Helmholtz equation. As mentioned in Section 2, in spherical coordinates the eigenfunctions are parameterized by two indices, $l$ and $m$, which take on integer values. The partial-wave index $l$ qualitatively describes the impact parameter distance of an incident ray from the origin of coordinates, and the azimuthal index $m$ is related to the angular clustering with respect to the origin of all the rays that have a given impact parameter. In cylindrical coordinates, the eigenfunctions are parameterized by the partial-wave index $l$, which now has integer values $-\infty<l<\infty$, which qualitatively describes the impact parameter distance of an incident ray to the left or right of the origin, and a continuous index $h$ with $-\infty<h<\infty$, which is related to the propagation direction $\eta$ of a plane-wave component in the angular spectrum of the incident beam with respect to the $x y$ plane. As a result, the cylinder partial-wave scattering amplitudes $a_{l}(h), b_{l}(h)$, and $q_{l}(h)$ are functions of $\eta$, whereas the sphere partial-wave scattering amplitudes $a_{l}$ and $b_{l}$ are independent of $m$.

It then follows that the relation between $\lambda, a$, and $n$ for the excitation of a cylinder MDR also depends ${ }^{31,32}$ on $\eta$. Specifically, for small $\eta$, the particle size parameter at resonance $x_{\text {res }}(\eta)$ is given approximately by

$$
x_{\mathrm{res}}(\eta) \approx x_{\mathrm{res}}(0)+K \eta^{2},
$$

where $K \approx L / 2 n^{3}$ for a low-radial-order MDR and $K$ grows to a few times this value for high-radial-order MDR's. The $\eta$ dependence of $x_{\text {res }}$ is illustrated in Fig. 2(a) and strongly influences the MDR excitation efficiency. This is so because the localized approximation beam-shape coefficients $A_{l}(h)$ and $B_{l}(h)$ [or $A_{l}(\eta)$ and $B_{l}(\eta)$, as we will write hereafter since $h \sim \sin \eta$ for the nonevanescent components in the interval $-1 \leqslant h \leqslant 1$ ] for a focused Gaussian beam expressed in cylindrical coordinates are based on modeling the beam as an angular spectrum of plane waves. ${ }^{33,34}$ Specifically, if the propagation direction of a Gaussian beam is normal to the fiber axis $(\xi=0)$, the component plane waves in its angular spectrum are incident on the fiber with a small range of angles $\Delta \eta=4 s$ centered about $\eta=0$ [see Fig. 2(b)]. Thus if $\lambda, a$, and $n$ are such that the $\eta=0$ component in the angular spectrum excites a MDR at the center of its linewidth [i.e., $x=x_{\text {res }}(0)$ ], other plane-wave components in the beam's angular spectrum correspond to values of $\eta$ for which $x \neq x_{\text {res }}(\eta)$. Either the size parameter $x$ is off the center of the resonance and excites it only partially or it lies beyond the MDR linewidth and fails to excite the resonance. We can see this by comparing Figs. 2(a) and 2(b), where the beam-shape coefficients are nonzero for a range of plane-wave components $\eta$ in which only the $\eta \approx 0$ components excite the resonance.

We consider a cylindrical fiber whose axis coincides with the $z$ axis, a Gaussian beam propagating parallel to the $x$ axis whose focal waist is located at $\left(0, y_{0}, 0\right)$, and a detector in the form of a circular cylinder of radius $r$ in the scattering far zone and concentric with the fiber axis. This geometry is shown in Fig. 3. Again we define the MDR excitation efficiencies $\epsilon_{L}^{\mathrm{TE}}$ and $\epsilon_{L}^{\mathrm{TM}}$ as the power elas- tically scattered by the TE-polarized or the TM-polarized MDR divided by the beam power. The power of an unscattered TE-polarized beam striking the detector in the interval $\Delta z$ of Fig. 3(b) is

$$
P_{\text {beam }}^{\mathrm{TE}}=\left(\frac{E_{0}^{2}}{2 \mu_{0} c}\right)\left(\frac{2 \pi}{k^{2} r}\right) \Delta z \cos ^{5} \eta \sum_{l=-\infty}^{\infty}\left|A_{l}(\eta)\right|^{2},
$$

and the power of an unscattered TM-polarized beam striking the detector is

$$
P_{\text {beam }}^{\mathrm{TM}}=\left(\frac{E_{0}^{2}}{2 \mu_{0} c}\right)\left(\frac{2 \pi}{k^{2} r}\right) \Delta z \cos ^{5} \eta \sum_{l=-\infty}^{\infty}\left|B_{l}(\eta)\right|^{2} .
$$

The localized approximation beam-shape coefficients, expressed in cylindrical coordinates for a normally incident TE-polarized beam with its beam-waist center at $\left(0, y_{0}, 0\right)$, are $^{33}$

$$
\begin{aligned}
A_{l}(\eta)= & \left(2 s \pi^{1 / 2} \cos ^{2} \eta\right)^{-1} \exp \left(-\sin ^{2} \eta / 4 s^{2}\right) \\
& \times \exp \left[-s^{2}\left(l / \cos \eta+k y_{0}\right)^{2}\right], \\
B_{l}(\eta)= & 0 .
\end{aligned}
$$

For a TM-polarized beam the expressions for $A_{l}(\eta)$ and $B_{l}(\eta)$ are interchanged. Notice that the localized beam coefficients for scattering by a sphere have the TE or TM nature of the polarization of the incident beam encoded into both $A_{l, m}$ and $B_{l, m}$ in Eqs. (7), whereas the polarization character of the incident beam for scattering by a cyl-

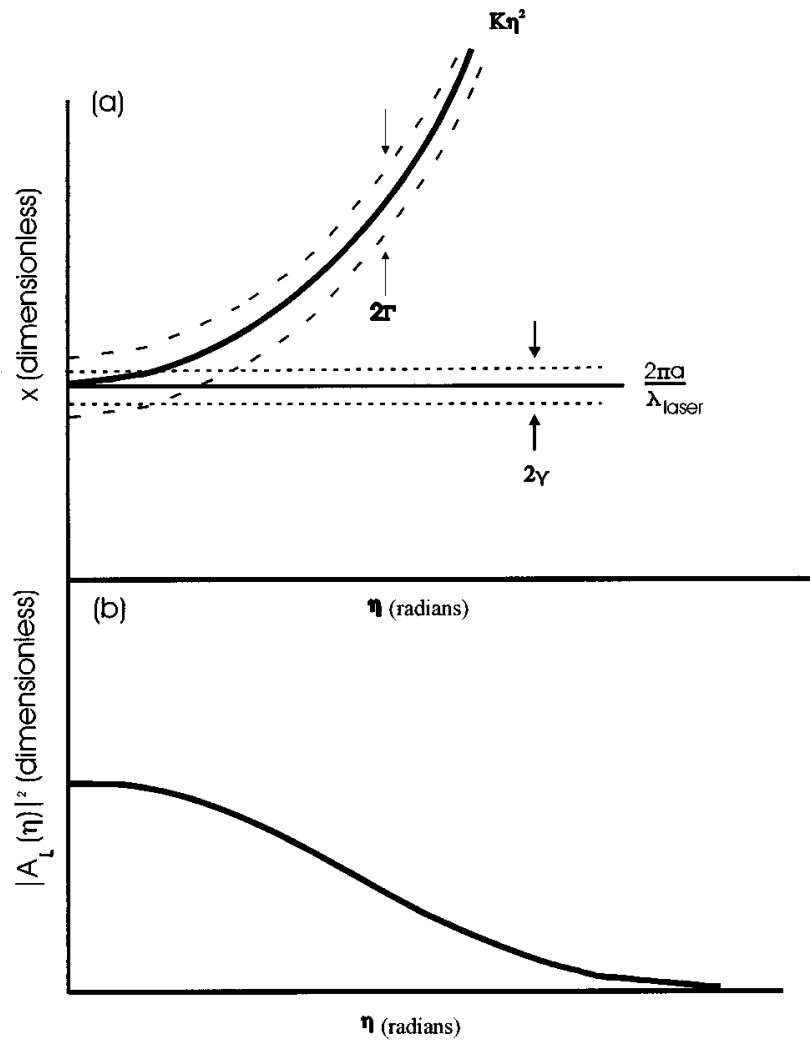

Fig. 2. Dependence of (a) the resonant size parameter and (b) the incident beam's angular spectrum of plane waves on the angle $\eta$ that a plane-wave component makes with the normal to the cylinder axis. The MDR linewidth is $2 \Gamma$, the laser beam linewidth is $2 \gamma$, and the beam's angular spectrum extends to $\eta$ $\approx 2 s$. 


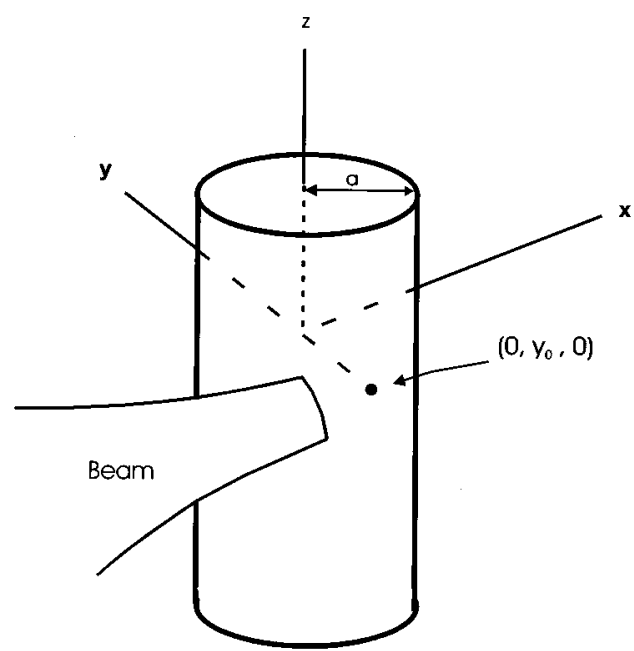

(a)

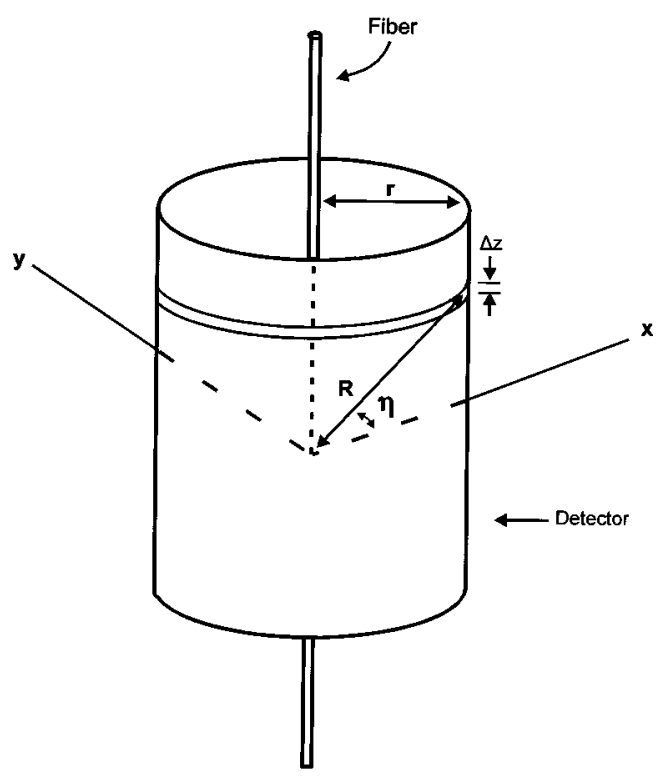

(b)

Fig. 3. (a) Focused Gaussian beam with the center of its focal waist at $\left(0, y_{0}, 0\right)$ normally incident on a cylindrical fiber of radius $a$ whose axis coincides with the $z$ axis. (b) Detector a distance $r$ from the fiber in the far zone and concentric with the fiber axis.

inder is encoded only in $A_{l}(\eta)$ or $B_{l}(\eta)$. When the integral over $z$ [or equivalently over $\eta$, since $z=R \sin \eta$ in Fig. 3(b)] on the detector is performed in relations (26) and (27), the beam power becomes

$$
P_{\text {beam }}=\left(\frac{E_{0}^{2}}{2 \mu_{0} c}\right)\left(\frac{\pi w_{0}^{2}}{2}\right),
$$

as expected.

The power of a TE-polarized incident beam that is elastically scattered by the fiber into the interval $\Delta z$ in Fig. $3(\mathrm{~b})$ is

$$
\begin{aligned}
P_{\mathrm{scatt}}^{\mathrm{TE}}= & \left(\frac{E_{0}{ }^{2}}{2 \mu_{0} c}\right)\left(\frac{8 \pi}{k^{2} r}\right) \Delta z \cos ^{5} \eta \sum_{l=-\infty}^{\infty}\left|A_{l}(\eta)\right|^{2} \\
& \times\left[\left|a_{l}(\eta)\right|^{2}+\left|q_{l}(\eta)\right|^{2}\right],
\end{aligned}
$$

and for a TM-polarized incident beam the scattered power is

$$
\begin{aligned}
P_{\mathrm{scatt}}^{\mathrm{TM}}= & \left(\frac{E_{0}{ }^{2}}{2 \mu_{0} c}\right)\left(\frac{8 \pi}{k^{2} r}\right) \Delta z \cos ^{5} \eta \sum_{l=-\infty}^{\infty}\left|B_{l}(\eta)\right|^{2} \\
& \times\left[\left|b_{l}(\eta)\right|^{2}+\left|q_{l}(\eta)\right|^{2}\right] .
\end{aligned}
$$

At normal incidence and for no absorption, the partialwave scattering amplitudes at the center of the linewidth of a TE-polarized MDR in the partial wave $L$ satisfy ${ }^{31}$ $\left|a_{L}(0)\right|=1.0$ and $\left|q_{L}(0)\right|=0.0$, and for a TM-polarized MDR they satisfy $\left|b_{L}(0)\right|=1.0$ and $\left|q_{L}(0)\right|=0.0$. Combining formulas (27)-(32) and considering the incident beam intensity and the scattered intensity only in the interval $\Delta z$ centered about $z=0$, we arrive at a normalincidence cylinder MDR excitation efficiency of

$$
\epsilon_{L}^{\mathrm{TE}} \approx \epsilon_{L}^{\mathrm{TM}} \approx\left(\frac{2}{\pi}\right)^{1 / 2}\left(\frac{4}{k w_{0}}\right) \exp \left[-2 s^{2}\left(L \pm k y_{0}\right)^{2}\right]
$$

This $z=0$ excitation efficiency reaches its maximum value

$$
\left(\epsilon_{L}\right)_{\max } \approx\left(\frac{2}{\pi}\right)^{3 / 2}\left(\frac{\lambda}{w_{0}}\right)
$$

when the beam is positioned off axis at the location

$$
k y_{0}= \pm L \text {. }
$$

Relation (34) for the $z=0$ efficiency is identical to the sphere MDR excitation efficiency of relation (24).

But when the $z$ (or equivalently the $\eta$ ) integral is performed for the scattered intensity in Eqs. (31) and (32), the result is significantly smaller than that for a sphere MDR for two reasons. First, as mentioned above, the magnitude of the partial-wave scattering amplitudes that appear in the integrand decrease rapidly from unity for $\eta \neq 0$ since the relation between $\lambda, a$, and $n$ at resonance for $\eta \neq 0$ differs from that for $\eta=0$. Thus, if the angular spectrum of the incident beam is sufficiently wide, a number of the $\eta \neq 0$ plane-wave components in the angular spectrum will not resonate. Second, according to Eqs. (29), the optimal ray impact parameter for MDR generation by a plane wave with an angle of incidence $\eta$ is

$$
k y_{0}= \pm L / \cos \eta \text {. }
$$

So if $y_{0}$ is such that the $\eta=0$ ray is at the optimal impact parameter, the $\eta \neq 0$ rays will not be.

Although MDR line shapes are Lorentzian, ${ }^{35}$ we model them here as Gaussians,

$$
\left|a_{L}(\eta)\right|^{2} \sim\left|b_{L}(\eta)\right|^{2} \approx \exp \left\{-\left[x-x_{\mathrm{res}}(\eta)\right]^{2} / \Gamma^{2}\right\},
$$

for calculational ease. We also assume that the crosspolarized scattering amplitude vanishes:

$$
\left|q_{L}(\eta)\right|^{2} \approx 0
$$

which is an accurate approximation for small $\eta$. Substituting Eqs. (29) and relation (37) into Eqs. (31) and (32) and integrating over $\eta$ for the single partial wave $L$, we find that the power scattered by the MDR becomes 


$$
\begin{aligned}
P_{L}^{\text {scatt }} \approx & \left(\frac{E_{0}^{2}}{2 \mu_{0} c}\right)\left(2 w_{0}^{2}\right) \int_{-\infty}^{\infty} \cos \eta \mathrm{d} \eta \\
& \times \exp \left[-2 s^{2} L^{2}\left(\frac{1}{\cos \eta}-1\right)^{2}\right] \\
& \times \exp \left(-\sin ^{2} \eta / 2 s^{2}\right) \\
& \times \exp \left\{-\left[x-x_{\text {res }}(0)-K \eta^{2}\right]^{2} / \Gamma^{2}\right\} .
\end{aligned}
$$

When $\lambda, a$, and $n$ satisfy $x=x_{\text {res }}(0)$ and the beam is optimally positioned as in Eq. (35), the power elastically scattered by the MDR mode simplifies to

$$
\begin{aligned}
P_{L}^{\text {scatt }} \approx & \left(\frac{E_{0}^{2}}{2 \mu_{0} c}\right)\left(2 w_{0}^{2}\right) \int_{-\infty}^{\infty} \mathrm{d} \eta \exp \left(-\eta^{2} / 2 s^{2}\right) \\
& \times \exp \left[-\left(\frac{s^{2} L^{2}}{2}+\frac{K^{2}}{\Gamma^{2}}\right) \eta^{4}\right],
\end{aligned}
$$

where $\exp \left(-s^{2} L^{2} \eta^{4} / 2\right)$ and $\exp \left(-K^{2} \eta^{4} / \Gamma^{2}\right)$ describe the dependence of the optimal ray impact parameter and the resonant size parameter, respectively, on $\eta$. The integral in relation (40) may be evaluated to give ${ }^{36}$

$$
P_{L}^{\text {scatt }} \approx\left(\frac{E_{0}^{2}}{2 \mu_{0} c}\right)\left(2 w_{0}^{2}\right) 2 s \Omega^{1 / 2} \exp (\Omega) K_{1 / 4}(\Omega),
$$

where $K_{1 / 4}(\Omega)$ is a modified Bessel function of the second kind and

$$
\Omega=\left(s^{2} L^{2}+2 K^{2} / \Gamma^{2}\right)^{-1}(2 s)^{-4} .
$$

The author is not aware of a simple method for approximating $K_{1 / 4}(\Omega)$, except in the $\Omega \rightarrow 0$ and $\Omega \rightarrow \infty$ limits. $^{37}$

If we ignore the $\eta$ dependence of the resonances, i.e., the $\exp \left(-\eta^{4}\right)$ terms, we have the $\Omega \rightarrow \infty$ limit, and $\epsilon_{L}$ reduces to the value given in relation (34). On the other hand, it usually happens that the MDR half-width $\Gamma$ is sufficiently narrow that the $\eta$ dependence of $x_{\text {res }}(\eta)$ is stronger than the $\eta$ dependence of the optimal ray impact parameter; i.e.,

$$
2 K^{2} / \Gamma^{2} \gg s^{2} L^{2}
$$

In addition, if the resonance is narrow and the beam is focused at least moderately, we also have

$$
\Gamma / K \ll 2 s^{2},
$$

and the $\exp \left(-\eta^{2} / 2 s^{2}\right)$ factor in relation (40) dies off much more slowly as a function of $\eta$ than does the $\exp \left(-K^{2} \eta^{4} / \Gamma^{2}\right)$ factor. We then have the $\Omega \rightarrow 0$ limit, and relation (41) becomes ${ }^{37,38}$

$$
\begin{aligned}
P_{L}^{\text {scatt }} & \approx\left(\frac{E_{0}^{2}}{2 \mu_{0} c}\right)\left(2 w_{0}^{2}\right) \int_{-\infty}^{\infty} \mathrm{d} \eta \exp \left(-K \eta^{4} / \Gamma^{2}\right) \\
& =\left(\frac{E_{0}^{2}}{2 \mu_{0} c}\right)\left(w_{0}^{2}\right)\left(\frac{\Gamma}{K}\right)^{1 / 2} \Gamma\left(\frac{1}{4}\right),
\end{aligned}
$$

where the gamma function $\Gamma(1 / 4) \approx 3.6256$ is not to be confused with the resonance half-width $\Gamma$. The MDR excitation efficiency then becomes

$$
\epsilon_{L}^{\mathrm{TE}}=\epsilon_{L}^{\mathrm{TM}}=\frac{2}{\pi}\left(\frac{\Gamma}{K}\right)^{1 / 2} \Gamma(1 / 4)
$$

which is independent of $w_{0}$ since the degree to which the cylinder MDR is excited is determined by the resonance half-width $\Gamma$ rather than by the number of rays that have the optimum impact parameter as was the case in Section 2 for sphere MDR's.

As a numerical example, we consider the same beam parameters that were employed in the numerical example of Section 2. A long fiber of radius $a=22.5 \mu \mathrm{m}$ and refractive index $n=1.5$ is illuminated by laser light of $\lambda$ $=0.5145 \mu \mathrm{m}$ and $w_{0}=8 \mu \mathrm{m}$ exciting a MDR in partial wave $L=300$ of half-width $\Gamma=5.3 \times 10^{-4}$ (corresponding to $\Delta \lambda_{\mathrm{MDR}}=10^{-3} \mathrm{~nm}$, which is a typical value). If the incident beam's focal waist is positioned so as to maximize the MDR excitation efficiency, we obtain from Eq. (46) $\epsilon_{300} \approx 0.6 \times 10^{-2}$, rather than the value $3.3 \times 10^{-2}$ that was obtained for a sphere MDR. If $\Gamma$ is smaller than the value given above, the excitation efficiency of the cylinder MDR decreases from the value quoted here. Again, the derivation leading to Eq. (46) ignored the finite linewidth of the laser. Including this effect, we obtain

$$
\epsilon_{L}^{\mathrm{TE}}=\epsilon_{L}^{\mathrm{TM}}=\frac{2}{\pi} \frac{\left(\Gamma^{2}+\gamma^{2}\right)^{1 / 4}}{K^{1 / 2}}\left[\frac{\Gamma}{\left(\Gamma^{2}+\gamma^{2}\right)^{1 / 2}}\right] \Gamma\left(\frac{1}{4}\right)
$$

at the optimal beam position in the $\Omega \rightarrow 0$ limit, instead of Eq. (46)

It would be of interest to perform an experiment in which a MDR is excited in either a spherical particle or a long test fiber in contact with a beam-carrying optical fiber $^{19}$ to verify the lower excitation efficiency of cylinder MDR's. It would also be of interest to measure the excitation efficiency as the angle between the long test fiber and the beam-carrying optical fiber was varied away from $90^{\circ}$ so that spiral MDR's would be excited in the test fiber.

\section{DISCUSSION}

The principal results of this study are formulas (16), (24), (40), and (46), which give the excitation efficiency of a morphology-dependent resonance produced by a focused Gaussian beam of arbitrary width and with the illuminated particle at an arbitrary position in the plane of the focal waist. The off-axis focal waist position that produces maximal efficiency is given by Eqs. (23) and (35). The physical interpretation and implications of these results have also been discussed. In this section their limitations are discussed.

The derivation of the excitation efficiency in this paper depended crucially on there being radiative equilibrium between the incident beam and the target particle. But radiative equilibrium is not necessarily achieved when the laser beam is pulsed. Nonetheless, all the photons from one pulse coupled into a MDR mode are eventually released back to the outside as scattered light, assuming that no absorption or inelastic processes occur. Thus one could redefine the excitation efficiency for a pulsed beam as the energy coupled into the MDR per pulse, or equivalently as the energy scattered by the MDR mode beginning when the pulse first encounters the particle at $t$ $=0$ and extending to $t \rightarrow \infty$, divided by the beam energy per pulse. 
When one takes into account the molecular composition of the target particle, all photons from either a cw or a pulsed beam coupled into a MDR mode are eventually absorbed, elastically scattered, or inelastically scattered. If the target particle is partially absorptive and the refractive index is complex, some of the energy coupled into the MDR is converted into heat or into phonons as the MDR wave orbits beneath the particle surface. One could extend the definition of the MDR excitation efficiency to include absorption by calculating the extinction rather than the elastically scattered power and by modeling the real part of the resonant partial-wave scattering amplitude as a Gaussian whose height at a MDR and whose width are functions of the imaginary part of the refractive index. ${ }^{39}$

The definition of the excitation efficiency need not be radically altered if an inelastic process such as fluorescence, lasing, or stimulated Raman scattering occurs. Inelastic processes that arise from molecular excitation cannot be treated exactly and completely in the context of Lorenz-Mie theory. ${ }^{40}$ Rather, the inelastic excitation rate must be calculated by use of quantum-mechanical perturbation theory, ${ }^{41}$ with the vector potential of the interior electric field as the perturbing potential. In many inelastic processes, however, each scattered photon that is created at the shifted frequency arises from the annihilation of a single photon at the original frequency. Thus the number of elastically plus inelastically scattered photons per second is equal to the number of elastically scattered photons per second calculated in Sections 2 and 3, assuming that no inelasticities occurred. The definition of the MDR excitation efficiency can then be recast in terms of the number of incoming and outgoing photons rather than in terms of incoming and outgoing power.

We found in Sections 2 and 3 that the scattering efficiency of a low-radial-order MDR is very small because the MDR linewidth is much narrower than the laser linewidth. The situation for the interior electric field for lowradial-order MDR's, however, is somewhat different. Even if the beam energy coupled to a low-radial-order MDR is quite low, a MDR photon can orbit beneath the particle surface for a very long time before finally escaping as scattered light owing to the high $Q$ of the resonance. The resulting interior energy density can thus grow quite large and can even dominate the total interior energy density. The dominance can be sufficiently great that a low-radial-order MDR will still provide the major contribution to the interior energy density hundreds of linewidths away from the center of the resonance. ${ }^{13}$

As a final thought, we have found that the localized beam-shape coefficients lead to a substantial mathematical simplification of the formulas that describe the excitation of a MDR by a focused beam, and they provide a pleasing physical picture of the excitation process. These results further attest to the fact that localized beam models represent a significant and useful addition to LorenzMie theory.

\section{ACKNOWLEDGMENT}

The author thanks Stephen Arnold for bringing this problem to his attention.
Note added in proof: Equation (26) has been recently obtained as the Taylor series expansion of a more general expression for the MDR shift in Ref. 42. The results of Eqs. (23) and (35) are not accurate for low-radial-order MDR's in slightly distorted microcavities. ${ }^{43}$

\section{REFERENCES AND NOTES}

1. H. C. van de Hulst, Light Scattering by Small Particles (Dover, New York, 1981), pp. 208-209.

2. H. M. Nussenzveig, "Tunneling effects in diffractive scattering and resonances," Comments At. Mol. Phys. 4, 175187 (1989).

3. L. G. Guimaraes and H. M. Nussenzveig, "Theory of Mie resonances and ripple fluctuations," Opt. Commun. 89, 363-369 (1992).

4. B. R. Johnson, "Theory of morphology-dependent resonances: shape resonances and width formulas," J. Opt. Soc. Am. A 10, 343-352 (1993).

5. P. R. Conwell, P. W. Barber, and C. K. Rushforth, "Resonant spectra of dielectric spheres," J. Opt. Soc. Am. A 1, 62-67 (1984).

6. P. Chylek, J. D. Pendleton, and R. G. Pinnick, "Internal and near-surface scattered field of a spherical particle at resonant conditions," Appl. Opt. 24, 3940-3942 (1985).

7. P. Chylek, "Partial-wave resonances and the ripple structure in the Mie normalized extinction cross section," J. Opt. Soc. Am. 66, 285-287 (1976).

8. A. Ashkin and J. M. Dziedzic, "Observation of optical resonances of dielectric spheres by light scattering," Appl. Opt. 20, 1803-1814 (1981).

9. T. Baer, "Continuous-wave laser oscillation in a Nd:YAG sphere," Opt. Lett. 12, 392-394 (1987).

10. J.-Z. Zhang, D. H. Leach, and R. K. Chang, "Photon lifetime within a droplet: temporal determination of elastic and stimulated Raman scattering," Opt. Lett. 13, 270-272 (1988).

11. B. Maheu, G. Gréhan, and G. Gouesbet, "Ray localization in Gaussian beams," Opt. Commun. 70, 259-262 (1989).

12. J. P. Barton, D. R. Alexander, and S. A. Schaub, "Internal fields of a spherical particle illuminated by a tightly focused laser beam: focal point positioning effects at resonance," J. Appl. Phys. 65, 2900-2906 (1989).

13. E. E. M. Khaled, S. C. Hill, P. W. Barber, and D. Q. Chowdhury, "Near-resonance excitation of dielectric spheres with plane waves and off-axis Gaussian beams," Appl. Opt. 31, 1166-1169 (1992).

14. E. E. M. Khaled, S. C. Hill, and P. W. Barber, "Internal electric energy in a spherical particle illuminated with a plane wave or off-axis Gaussian beam," Appl. Opt. 33, 524532 (1994)

15. J. A. Lock, "Improved Gaussian beam-scattering algorithm," Appl. Opt. 34, 559-570 (1995).

16. G. Gouesbet, G. Gréhan, and B. Maheu, "Localized interpretation to compute all the coefficients $g_{n m}$ in the generalized Lorenz-Mie theory," J. Opt. Soc. Am. A 7, 998-1007 (1990).

17. J. A. Lock and G. Gouesbet, "Rigorous justification of the localized approximation to the beam-shape coefficients in generalized Lorenz-Mie theory. I. On-axis beams," J. Opt. Soc. Am. A 11, 2503-2515 (1994).

18. G. Gouesbet and J. A. Lock, "Rigorous justification of the localized approximation to the beam-shape coefficients in generalized Lorenz-Mie theory. II. Off-axis beams," J. Opt. Soc. Am. A 11, 2516-2525 (1994).

19. A. Serpenguzel, S. Arnold, G. Griffel, and J. A. Lock, "Enhanced coupling to microsphere resonances with optical fibers," J. Opt. Soc. Am. B 14, 790-795 (1997).

20. Equation (63) of Ref. 15 giving the optimal beam position for sphere MDR's, is also valid for cylinder MDR's since the calculation leading to Eq. (1.1) of C. C. Lam, P. T. Leung, and K. Young, "Explicit asymptotic formulas for the positions, widths, and strengths of resonances in Mie scattering," J. Opt. Soc. Am. B 9, 1585-1592 (1992), on which the 
result of Ref. 15 is based, is valid for Bessel functions of both integer order (cylinders) and half-integer order (spheres).

21. G. Gouesbet, B. Maheu, and G. Gréhan, "Light scattering from a sphere arbitrarily located in a Gaussian beam, using a Bromwich formulation,” J. Opt. Soc. Am. A 5, 1427-1443 (1988).

22. A. Gray and G. B. Mathews, A Treatise on Bessel Functions and Their Applications to Physics (Dover, New York, 1966), p. 36

23. I. S. Gradshteyn and I. M. Ryzhik, Table of Integrals, Series, and Products (Academic, New York, 1965), p. 718, Eq. (6.633.4).

24. H. Kogelnik and T. Li, "Laser beams and resonators," Appl. Opt. 5, 1550-1567 (1966).

25. M. Abramowitz and I. A. Stegun, Handbook of Mathematical Functions (National Bureau of Standards, Washington, D.C., 1964), p. 378, Eqs. (9.8.1)-(9.8.4) along with the recursion relation of Eq. (9.6.26).

26. M. Abramowitz and I. A. Stegun, Handbook of Mathematical Functions (National Bureau of Standards, Washington, D.C., 1964), p. 375, Eq. (9.6.10).

27. M. Abramowitz and I. A. Stegun, Handbook of Mathematical Functions (National Bureau of Standards, Washington, D.C., 1964), p. 377, Eq. (9.7.1).

28. P. Chylek, J. T. Kiehl, and M. K. W. Ko, "Optical levitation and partial wave resonances," Phys. Rev. A 18, 2229-2233 (1978).

29. P. M. Aker, P. A. Moortgat, and J.-X. Zhang, "Morphologydependent stimulated Raman scattering imaging. 1. Theoretical aspects," J. Chem. Phys. 105, 7268-7275 (1996).

30. C. F. Bohren and D. R. Huffman, Absorption and Scattering of Light by Small Particles (Wiley, New York, 1983), pp. 194-204.

31. J. A. Lock, "Morphology-dependent resonances of an infinitely long circular cylinder illuminated by a diagonally incident plane wave or a focused Gaussian beam," J. Opt. Soc. Am. A 14, 653-661 (1997).

32. L. G. Guimaraes, J. P. Rodrigues, and F. de Mendonca,
"Analysis of the resonant scattering of light by cylinders at oblique incidence," Appl. Opt. 36, 8010-8019 (1997).

33. J. A. Lock, "Scattering of a diagonally incident focused Gaussian beam by an infinitely long homogeneous circular cylinder," J. Opt. Soc. Am. A 14, 640-652 (1997).

34. Another localized approximation for the beam-shape coefficients for scattering by a cylinder is described in K. F. Ren, G. Gréhan, and G. Gouesbet, "Scattering of a Gaussian beam by an infinite cylinder in the framework of generalized Lorenz-Mie theory: formulation and numerical results," J. Opt. Soc. Am. A 14, 3014-3025 (1997).

35. S. C. Hill and R. E. Benner, "Morphology-dependent resonances," in Optical Effects Associated with Small Particles, P. W. Barber and R. K. Chang, eds. (World Scientific, Singapore, 1988), pp. 3-61.

36. I. S. Gradshteyn and I. M. Ryzhik, Table of Integrals, Series, and Products (Academic, New York, 1965), p. 307, Eq (3.323.3).

37. M. Abramowitz and I. A. Stegun, Handbook of Mathematical Functions (National Bureau of Standards, Washington, D.C., 1964), p. 375, Eq. (9.6.9); p. 378, Eq. (9.7.2).

38. I. S. Gradshteyn and I. M. Ryzhik, Table of Integrals, Series, and Products (Academic, New York, 1965), p. 307, Eq (3.326).

39. M. Kerker, The Scattering of Light and Other Electromagnetic Radiation (Academic, New York, 1969), pp. 98-99, Figs. 4.1 and 4.2 .

40. The situation is described clearly in M. Kerker, P. J. McNulty, M. Sculley, H. Chew, and D. D. Cooke, "Raman and fluorescent scattering by molecules embedded in small particles: numerical results for incoherent optical processes," J. Opt. Soc. Am. 68, 1676-1686 (1978).

41. J. J. Sakurai, Advanced Quantum Mechanics (AddisonWesley, Reading, Mass., 1980), pp. 36-57.

42. G. Roll and G. Schweiger, "Resonance shift of obliquely illuminated dielectric cylinders," Appl. Opt. 37, 5628-5630 (1998)

43. H.-B. Lin, J. D. Eversole, A. J. Campillo, and J. P. Barton, "Excitation localization principle for spherical microcavities," Opt. Lett. (to published). 